Article

\title{
Candidate Genes Involved in Beneficial or Adverse Responses to Commonly Eaten Brassica Vegetables in a New Zealand Crohn's Disease Cohort
}

\author{
Bobbi Laing *, Dug Yeo Han and Lynnette R. Ferguson \\ Discipline of Nutrition, School of Medical Sciences, Auckland University, 85 Park Road, \\ Grafton Campus, Auckland 1142, New Zealand; E-Mails: dy.han@auckland.ac.nz (D.Y.H.); \\ 1.ferguson@auckland.ac.nz (L.R.F.) \\ * Author to whom correspondence should be addressed; E-Mail: b.laing@auckland.ac.nz; \\ Tel.: +64-9-923-1785; Fax: +64-9-923-3362.
}

Received: 4 September 2013; in revised form: 22 November 2013 / Accepted: 29 November 2013 / Published: 12 December 2013

\begin{abstract}
Crohn's disease (CD) is one of the two manifestations of inflammatory bowel disease. Particular foods are thought with $\mathrm{CD}$ to exacerbate their illness. Vegetables, especially Brassicaceae, are often shunned by people with $\mathrm{CD}$ because of the negative effects they are alleged to have on their symptoms. Brassicaceae supply key nutrients which are necessary to meet recommended daily intakes. We sought to identify the candidate genes involved in the beneficial or adverse effects of Brassicaceae most commonly eaten, as reported by the New Zealand adults from the "Genes and Diet in Inflammatory Bowel disease Study" based in Auckland. An analysis of associations between the single nucleotide polymorphisms (SNPs) and the beneficial or adverse effects of the ten most commonly eaten Brassicaceae was carried out. A total of 37 SNPs were significantly associated with beneficial effects $(p=0.00097$ to 0.0497$)$ and 64 SNPs were identified with adverse effects ( $p=0.0000751$ to 0.049). After correcting for multiple testing, rs7515322 (DIO1) and rs9469220 (HLA) remained significant. Our findings show that the tolerance of some varieties of Brassicaceae may be shown by analysis of a person's genotype.
\end{abstract}

Keywords: Brassicaceae; tolerability; Crohn's disease; DIO1; HLA 


\begin{abstract}
Abbreviations
CD, Crohn's Disease; IBD, inflammatory bowel disease; NZ, New Zealand; SNP, Single nucleotide polymorphism; UC, Ulcerative Colitis.
\end{abstract}

\title{
1. Introduction
}

Crohn's disease (CD) is one of two commonly identified inflammatory bowel diseases (IBD) the other being ulcerative colitis (UC). Both disorders are inflammatory and people experience phases of remission and deterioration.

The incidence of CD seems to be increasing and it appears that New Zealand (NZ) in 2006 had the highest rate at $16.5 / 10^{5}$ [1]. Increases are observed particularly in the western world, and research has shown that the environment plays a considerable role [2]. It has been observed that individuals from Bangladesh (where the incidence of IBD is very low) on shifting to the United Kingdom, develop a very high incidence of IBD within a generation [3]. Nutrition is thought to play a key role and is considered to be an important environmental factor influencing the development of $\mathrm{CD}$ and its symptoms [4], through the nutrigenomic and epigenetic modification of the susceptibility genes [5].

Vegetables, especially Brassicaceae, are often shunned by people with CD because of the negative effects they are alleged to have on their symptoms [6,7]. However, Brassicaceae supply key nutrients. Some varieties of Brassicaceae also appear to be well tolerated by people with CD [8]. Several studies show that Brassicaceae contain many significant nutrients: fibre, the antioxidants vitamin $\mathrm{A}$ and $\mathrm{C}$, the vitamin folate and vitamin $\mathrm{K}$ and the minerals such as potassium, calcium, selenium and zinc as well as the numerous phytochemicals which have key roles in maintaining health [9-12]. They help improve immunity as well as contributing to anti-inflammatory and anti-cancer activities of the body [13-21]. The intake of sufficient amounts of these nutrients is important for people with CD. These nutrients are necessary to meet the daily intakes as recommended in the Nutrient Reference Values for Australia and New Zealand [22].

By studying the interaction of different Brassicaceae varieties with single nucleotide polymorphisms (SNPs) in people with $\mathrm{CD}$, it may be possible to uncover a genetic basis for individual tolerances. This could lead to more specific nutrition advice with respect to these important vegetables, and enhance the opportunities for CD patients to avail themselves of the benefits of Brassicaceae. The aim of this study was therefore to identify the candidate genes involved in the beneficial or adverse effects of the Brassicaceae most commonly eaten as reported by NZ adults from the "Genes and Diet in IBD Study"[4], based in Auckland.

\section{Materials and Methods}

\subsection{Brassicaceae Selection}

The Brassicaceae analysed for tolerability were those reported to be consumed by the subjects in the "Genes and Diet in IBD Study" based in Auckland NZ. These Brassicaceae were: broccoli, cabbage, cauliflower, Chinese greens, rocket (arugula), watercress, horseradish, mustard sauce, mustard powder and wasabi. 


\subsection{Tolerability of Brassicaceae}

The tolerability of the Brassicaceae was ascertained from secondary analysis of the information gained from the responses to the dietary questionnaire. This questionnaire was based on Joachim's methodology [23] and feedback from a group of patients with CD [4]. Questions in the survey were asked (and scored on a five-point scale on whether the person's IBD condition became either: definitely better, $(++)$ probably better $(+)$, had no effect $(=)$, probably worse $(-)$, and definitely worse ( -- in response to the listed food. This allowed information to be collected on how food affected their disease symptoms. This takes into account that many foods are never consumed because they are not considered palatable irrespective of the effects they are perceived to have on their symptoms of CD.

Two scales were used: the percentage of beneficial effects (definitely better and probably better) and adverse effects (definitely worse and probably worse). The responses to "makes no difference" were omitted. A nutritionist or registered nurse checked out the responses with respondents when there were any queries [4]. The dietary questionnaire also included open ended questions. These were also evaluated for additional information on foods which may affect symptoms associated with CD.

\subsection{Study Population}

Study participants were from the main North Island centre, Auckland and other major North Island centres. They were enrolled as part of a population based study in the IBD project whose purpose was to determine the genetic and environmental factors of CD aetiology. These people were enlisted from gastroenterology clinics or by their response from advertising in the media between May 2005 and April 2009. A total of 339 Caucasian subjects gave their informed consent to take part. The nutritional questionnaire was completed by 290 and up to 323 were genotyped (Table 1). Consent was obtained before the collection of peripheral blood for DNA extraction and genotyping. The questionnaires were taken away for participants to complete and on their return scrutinised for accuracy and completion and where necessary subjects were contacted again for clarification. All of the participants were selected on the basis of self-disclosed Caucasian ancestry only [4]. Clinical data (age, IBD diagnosis and the most recent Montreal classification of CD location [24]) were extracted from the clinical questionnaire and patient medical notes, which were supplied by the diagnosing gastroenterologist in the primary "Genes and Diet in IBD Study". Ethical approval was given by the NZ Multi-region Human Ethics Committee (MEC04/12/11).

\subsection{Genotyping}

SNP data with respect to the beneficial or adverse effects of selected Brassicaceae were taken from the original "Genes and Diet in IBD Study" based in Auckland, NZ. Gene polymorphisms in this study were determined by polymerase chain reaction-restriction fragment length polymorphism (PCR-RFLP) analysis. The SNPs identified for each Brassicaceae variety were corrected for multiple testing using a false discovery rate [25]. $\mathrm{R}$ was used for statistical analyses. A $p$-value was considered significant if it was less than 0.05 [26]. 
Table 1. Summary of sample sizes and clinical data for those with Crohn's disease (CD).

\begin{tabular}{ccc}
\hline Sub-phenotypes & $\begin{array}{c}\text { Phenotype } \\
\text { Description }\end{array}$ & $\boldsymbol{N}(\%)$ \\
\hline Age at diagnosis & $<17$ & $31(11.0)$ \\
Behaviour & $17-40$ & $205(72.7)$ \\
& $>40$ & $46(16.3)$ \\
& Inflammatory & $158(56.0)$ \\
& Stricturing & $91(32.3)$ \\
Location & Penetrating & $33(11.7)$ \\
& Ileal & $111(39.4)$ \\
& Colonic & $92(32.6)$ \\
Bowel Resection & Ileocolon & $79(28.0)$ \\
& No & $180(63.8)$ \\
& Yes & $102(36.2)$ \\
& No & $232(82.3)$ \\
Extra-intestinal manifestations & Yes & $50(17.7)$ \\
\hline
\end{tabular}

Note: A total of 339 participants were available for genotyping and up to 323 were genotyped. Of those, clinical information was available for 282 and the nutrition questionnaire was completed by 290 ; $N$-sample size.

\section{Results}

Table 2 shows the candidate genes. Table 3 shows the SNPs that were significantly associated with beneficial effects while Table 4 shows the significant SNPs which were associated with adverse effects on the symptoms of people with CD. Table S1 shows the Brassicacea frequencies $[n(\%)]$ by SNPS. A total of 37 variants were identified as having beneficial effects ( $p$ values from 0.00097 to 0.0497 ) and 64 SNPs with adverse effects (0.0000751 to 0.049). After correcting for multiple testing, two SNPs in two genes remained significant with adverse reactions to Brassicaceae. The rs7515322 variant in DIO1 was associated with Broccoli, and the rs9469220 variant in HLA was associated with rocket. These $p$-values were marked with an asterisk (*) (Table 4).

Table 2. CD related single nucleotide polymorphisms (SNPs) for association with Brassicaceae.

\begin{tabular}{cllc}
\hline Gene & \multicolumn{1}{c}{ Name } & \multicolumn{1}{c}{ SNP } & Chr \\
\hline DIO1 & deiodinase, iodothyronine, type I & rs11206244 & 1 \\
& & rs7515322 & 1 \\
IL23R & interleukin 23 receptor & rs11209026 & 1 \\
& interleukin 23 receptor & rs7517847 & 1 \\
ITLN1 & intelectin (galactofuranose binding) & rs2274910 & 1 \\
SEP15 & 15 kDa selenoprotein & rs5845 & 1 \\
& 15 kDa selenoprotein & rs5859 & 1 \\
TNFRSF1B & tumor necrosis factor receptor superfamily, member 1B & rs3397 & 1 \\
TNFSF15 & tumor necrosis factor (ligand) superfamily, member 15 & rs7029554 & 1 \\
ATG16L1 & ATG16 autophagy related 16-like 1 & rs10210302 & 2 \\
SLC11A1 & solute carrier family 11 (proton-coupled divalent metal ion & rs3731865 & 2 \\
\hline
\end{tabular}


Table 2. Cont.

\begin{tabular}{|c|c|c|c|}
\hline \multirow[t]{2}{*}{$A M T$} & aminomethyltransferase & rs11922013 & 3 \\
\hline & Aminomethyltransferase & rs1464567 & 3 \\
\hline \multirow[t]{2}{*}{$B S N$} & bassoon presynaptic cytomatrix & rs2131109 & 3 \\
\hline & bassoon presynaptic cytomatrix & rs4283605 & 3 \\
\hline$C D K A L 1$ & CDK5 regulatory subunit & rs6908425 & 3 \\
\hline FHIT & fragile histidine triad gene & rs2250114 & 3 \\
\hline$G P X 1$ & glutathione peroxidase 1 & rs1800668 & 3 \\
\hline \multirow[t]{2}{*}{ SLC6A6 } & $\begin{array}{l}\text { solute carrier family } 6 \text { (neurotransmitter transporter, } \\
\text { taurine), member } 6\end{array}$ & rs41284011 & 3 \\
\hline & $\begin{array}{l}\text { solute carrier family } 6 \text { (neurotransmitter transporter, } \\
\text { taurine), member } 6\end{array}$ & rs4685154 & 3 \\
\hline$T F$ & transferrin & rs1799899 & 3 \\
\hline TLRO & toll-like receptor 9 & rs5743836 & 3 \\
\hline \multirow[t]{4}{*}{ TRAIP } & TRAF interacting protein & rs10865959 & 3 \\
\hline & TRAF interacting protein & rs17598137 & 3 \\
\hline & TRAF interacting protein & rs2271960 & 3 \\
\hline & TRAF interacting protein & rs6446298 & 3 \\
\hline \multirow[t]{2}{*}{ USP4 } & ubiquitin specific peptidase 4 (proto-oncogene) & rs1865741 & 3 \\
\hline & ubiquitin specific peptidase 4 (proto-oncogene) & rs9881860 & 3 \\
\hline $\mathrm{CDH} 29$ & cadherin-related family member 4 & rs7629936 & 4 \\
\hline$A T G 12$ & ATG12 autophagy related 12 homolog (S. cerevisiae) & rs26532 & 5 \\
\hline CSF1R & colony stimulating factor 1 receptor & rs2282804 & 5 \\
\hline desert_PTGER4 & desert Prostaglandin E receptor 4 (subtype EP4) & rs17234657 & 5 \\
\hline desert_PTGER4 & desert Prostaglandin E receptor 4 (subtype EP4) & rs9292777 & 5 \\
\hline \multirow[t]{8}{*}{$G P X 3$} & glutathione perosidase 3 (plasma) & rs2042235 & 5 \\
\hline & glutathione perosidase 3 (plasma) & rs3763013 & 5 \\
\hline & glutathione perosidase 3 (plasma) & rs3792796 & 5 \\
\hline & glutathione perosidase 3 (plasma) & rs3792797 & 5 \\
\hline & glutathione perosidase 3 (plasma) & rs3805435 & 5 \\
\hline & glutathione perosidase 3 (plasma) & rs3828599 & 5 \\
\hline & glutathione perosidase 3 (plasma) & rs8177425 & 5 \\
\hline & glutathione perosidase 3 (plasma) & rs870407 & 5 \\
\hline IBD5 & inflammatory bowel disease & rs10077785 & 5 \\
\hline $\operatorname{IL} 12 B$ & interleukin 12B (natural killer cell stimulatory factor 2) & rs6887695 & 5 \\
\hline$I R G M$ & immunity-related GTPase family, & rs4958847 & 5 \\
\hline OCTN1/SLC22A4 & $\begin{array}{l}\text { solute carrier family } 22 \text { (organic cation/ergothioneine } \\
\text { transporter), member }\end{array}$ & rs1050152 & 5 \\
\hline OCTN2/SLC22A5 & $\begin{array}{l}\text { solute carrier family } 22 \text { (organic cation/carnitine } \\
\text { transporter), member } 5\end{array}$ & rs2631367 & 5 \\
\hline P4HA2 & prolyl 4-hydroxylase, alpha polypeptide II & rs4361509 & 5 \\
\hline \multirow[t]{2}{*}{ PTGER4 } & prostaglandin E receptor 4 (subtype EP4) & rs 1373692 & 5 \\
\hline & prostaglandin E receptor 4 (subtype EP4) & rs4613763 & 5 \\
\hline SEPP1 & selenoprotein $\mathrm{P}$, plasma, 1 & rs3877899 & 5 \\
\hline$H L A$ & major histocompatibility complex, class II, DO alpha & rs9469220 & 6 \\
\hline TNFALPHA & tumor necrosis factor & rs1800629 & 6 \\
\hline
\end{tabular}


Table 2. Cont.

\begin{tabular}{|c|c|c|c|}
\hline \multirow[t]{2}{*}{ CLDN12 } & claudin 12 & rs1017106 & 7 \\
\hline & claudin 12 & rs17864006 & 7 \\
\hline CNTNAP2 & contactin associated protein-like 2 & rs7807268 & 7 \\
\hline IL6 & interleukin 6 (interferon, beta 2) & rs 1800795 & 7 \\
\hline DEFA6 & defensin, alpha 6 , Paneth cell-specific & rs712276 & 8 \\
\hline$J A K 2$ & janus kinase 2 & rs10758669 & 9 \\
\hline \multirow[t]{3}{*}{ TNFSF 15} & tumor necrosis factor (ligand) superfamily, member 15 & rs10982412 & 9 \\
\hline & tumor necrosis factor (ligand) superfamily, member 15 & rs3810936 & 9 \\
\hline & tumor necrosis factor (ligand) superfamily, member 15 & rs7867918 & 9 \\
\hline$D L G 5$ & discs, large homolog 5 (Drosophila) & rs2289311 & 10 \\
\hline$N K X 2-3$ & NK2 homeobox 3 & rs 10883365 & 10 \\
\hline$J A M 3$ & junctional adhesion molecule 3 & rs11604455 & 11 \\
\hline \multirow[t]{2}{*}{$S L C 11 A 2$} & $\begin{array}{l}\text { solute carrier family } 11 \text { (proton-coupled divalent metal ion } \\
\text { transporters), member } 2\end{array}$ & rs224589 & 12 \\
\hline & $\begin{array}{l}\text { solute carrier family } 11 \text { (proton-coupled divalent metal ion } \\
\text { transporters), member } 3\end{array}$ & rs427020 & 12 \\
\hline$V D R$ & vitamin D (1,25- dihydroxyvitamin D3) receptor & rs7975232 & 12 \\
\hline$L A M P 1$ & lysosomal-associated membrane protein 1 & rs 12871648 & 13 \\
\hline $\mathrm{DIO} 2$ & deiodinase, iodothyronine, type II & rs 12885300 & 14 \\
\hline \multirow{8}{*}{ GPX2|CHURC1-FNTB } & deiodinase, iodothyronine, type III & rs1190715 & 14 \\
\hline & deiodinase, iodothyronine, type III & rs 1190716 & 14 \\
\hline & deiodinase, iodothyronine, type III & rs945006 & 14 \\
\hline & glutathione peroxidase 2, CHURC1-FNTB readthrough & rs 1800669 & 14 \\
\hline & glutathione peroxidase 2, CHURC1-FNTB readthrough & rs 2296327 & 14 \\
\hline & glutathione peroxidase 2, CHURC1-FNTB readthrough & rs 2412065 & 14 \\
\hline & glutathione peroxidase 2, CHURC1-FNTB readthrough & rs2737844 & 14 \\
\hline & glutathione peroxidase 2, CHURC1-FNTB readthrough & rs3742599 & 14 \\
\hline \multirow[t]{2}{*}{ SELS } & selenoprotein $\mathrm{S}$ & rs4965814 & 15 \\
\hline & selenoprotein $\mathrm{S}$ & rs7178239 & 15 \\
\hline$F A M 92 B$ & family with sequence similarity 92, member B & rs8050910 & 16 \\
\hline \multirow[t]{4}{*}{$M A P 1 L C 3 B$} & microtubule-associated protein 1 light chain 3 beta & rs2288483 & 16 \\
\hline & microtubule-associated protein 1 light chain 3 beta & rs 7204722 & 16 \\
\hline & microtubule-associated protein 1 light chain 3 beta & rs 8044820 & 16 \\
\hline & microtubule-associated protein 1 light chain 3 beta & rs 8051218 & 16 \\
\hline NOD2 & nucleotide-binding oligomerization domain containing 2 & rs 2066844 & 16 \\
\hline STAT3 & signal transducer and activator of transcription 3 & rs744166 & 17 \\
\hline$T N R C 6 C$ & trinucleotide repeat containing $6 \mathrm{C}$ & rs4362447 & 17 \\
\hline PTPN2 & protein tyrosine phosphatase, non-receptor type 2 & rs2542151 & 18 \\
\hline ICAMI & intercellular adhesion molecule 1 & rs1799969 & 19 \\
\hline$T F F 3$ & trefoil factor 3 (intestinal) & rs225369 & 21 \\
\hline$M I F$ & macrophage migration inhibitory factor & rs755622 & 22 \\
\hline$C L D N 2$ & claudin 2 & rs12008279 & $\mathrm{X}$ \\
\hline
\end{tabular}


Table 3. Results of SNPs from the candidate genes associated with beneficial effects of Brassicaceae; showing $p$-values only; significant $p$ values highlighted in italic and bold; n/r: no response.

\begin{tabular}{|c|c|c|c|c|c|c|c|c|c|c|}
\hline Gene & SNP & Cauliflower & Broccoli & Cabbage & Chinese Greens & rocket & Watercress & Mustard powder & Mustard Sauce & Wasabi \\
\hline$A M T$ & rs 11922013 & 0.2451 & 0.7510 & 0.3506 & 0.5299 & 0.6430 & 0.0418 & 0.3177 & 0.0172 & 0.9995 \\
\hline ATG12 & rs26532 & 0.0653 & 0.3809 & 0.3724 & 0.1532 & 0.0128 & 0.9788 & 0.0237 & 0.1822 & 0.0642 \\
\hline $\mathrm{CDH} 29$ & rs7629936 & 0.7431 & 0.7083 & 0.0454 & 0.3585 & 0.9900 & 0.3356 & 0.1522 & 0.4627 & 0.8607 \\
\hline CNTNAP2 & rs7807268 & 0.0899 & 0.4994 & 0.2787 & 0.0226 & 0.3516 & 0.1203 & 0.3510 & 0.1390 & 0.9995 \\
\hline CSF1R & rs2282804 & 0.0139 & 0.3442 & 0.4589 & 0.1351 & 0.6996 & 0.5417 & 0.9999 & 0.9441 & 0.9996 \\
\hline DEFA6 & rs712276 & 0.00097 & 0.0016 & 0.0048 & 0.0094 & 0.0302 & 0.0197 & 0.0310 & 0.7953 & 0.7298 \\
\hline desert_PTGER4 & rs9292777 & $\mathrm{n} / \mathrm{r}$ & $\mathrm{n} / \mathrm{r}$ & $\mathrm{n} / \mathrm{r}$ & 0.0296 & $\mathrm{n} / \mathrm{r}$ & $\mathrm{n} / \mathrm{r}$ & 0.9772 & 0.2711 & $\mathrm{n} / \mathrm{r}$ \\
\hline FAM92B & rs8050910 & 0.9996 & 0.0410 & 0.9995 & 0.1001 & 0.2675 & 0.9996 & 0.9999 & 0.9999 & 1.0000 \\
\hline FHIT & rs2250114 & 0.7337 & 0.8403 & 0.0590 & 0.9747 & 0.0508 & 0.7876 & 0.0354 & 0.0226 & 0.0507 \\
\hline$G P X 3$ & rs3763013 & 0.0103 & 0.0169 & 0.9996 & 0.2862 & 0.2754 & 0.2866 & 0.4011 & 0.6294 & 0.4772 \\
\hline$G P X 3$ & rs3792796 & 0.0187 & 0.0280 & 0.8501 & 0.3827 & 0.9010 & 0.6353 & 0.9240 & 0.2130 & 0.5985 \\
\hline$G P X 3$ & rs870407 & 0.9996 & 0.0475 & 0.7659 & 0.9368 & 0.7542 & 0.9859 & 0.8520 & 0.9999 & 0.9996 \\
\hline ICAMI & rs1799969 & 0.9168 & 0.9046 & 0.0270 & 0.8428 & 0.0060 & 0.0143 & 0.0138 & 0.2538 & 0.0635 \\
\hline IL6 & rs1800795 & 0.9363 & 0.5219 & 0.6916 & 0.8112 & 0.0046 & 0.4276 & 0.0744 & 0.0293 & 0.9996 \\
\hline$I R G M$ & rs4958847 & 0.1783 & 0.4637 & 0.2814 & 0.0378 & 0.5938 & 0.8985 & 0.8482 & 0.8803 & 0.4834 \\
\hline ITLN1 & rs2274910 & 0.3727 & 0.0374 & 0.6161 & 0.4158 & 0.5626 & 0.8039 & 0.3690 & 0.6410 & 0.7704 \\
\hline$J A M 3$ & rs 11604455 & 0.7261 & 0.0350 & 0.9996 & 0.8063 & 0.9997 & 0.9799 & 0.9999 & 0.9999 & 0.9997 \\
\hline$M A P 1 L C 3 B$ & rs 2288483 & 0.0403 & 0.0111 & 0.0590 & 0.2511 & 0.3290 & 0.5593 & 0.1842 & 0.9373 & 0.6128 \\
\hline$M A P 1 L C 3 B$ & rs7204722 & 0.2386 & 0.0361 & 0.3033 & 0.3514 & 0.5558 & 0.8065 & 0.3161 & 0.7094 & 0.7709 \\
\hline$M A P 1 L C 3 B$ & rs 8044820 & 0.5899 & 0.8822 & 0.0223 & 0.5065 & 0.8666 & 0.4233 & 0.4110 & 0.9999 & 0.9997 \\
\hline$M I F$ & rs755622 & 0.0139 & 0.0047 & 0.2191 & 0.3475 & 0.3667 & 0.5391 & 0.9999 & 0.2737 & 1.0000 \\
\hline$N K X 2.3$ & rs 10883365 & 0.0269 & 0.1211 & 0.1447 & 0.0157 & 0.0552 & 0.0225 & 0.0964 & 0.1120 & 0.9195 \\
\hline NOD2 & rs2066844 & 0.8928 & 0.6265 & 0.8517 & 0.9735 & 0.4115 & 0.9820 & 0.0397 & 0.9999 & 0.9996 \\
\hline PTGER4 & rs1373692 & 0.1842 & 0.0890 & 0.9742 & 0.0112 & 0.4711 & 0.5627 & 0.9594 & 0.3113 & 0.4380 \\
\hline$P T P N 2$ & rs 2542151 & 0.0103 & 0.0019 & 0.0023 & 0.0286 & 0.1077 & 0.0090 & 0.9999 & 0.9858 & 0.9168 \\
\hline
\end{tabular}


Table 3. Cont.

\begin{tabular}{|c|c|c|c|c|c|c|c|c|c|c|}
\hline$S E P \_15$ & rs5859 & 0.6706 & 0.1994 & 0.0535 & 0.4249 & 0.3915 & 0.7017 & 0.0497 & 0.5820 & 0.9369 \\
\hline SEPP1 & rs3877899 & 0.0045 & 0.0127 & 0.7790 & 0.1128 & 0.7454 & 0.0360 & 0.7790 & 0.6607 & 0.5572 \\
\hline SLC6AG & rs41284011 & $\mathrm{n} / \mathrm{r}$ & $\mathrm{n} / \mathrm{r}$ & 0.0094 & $\mathrm{n} / \mathrm{r}$ & $\mathrm{n} / \mathrm{r}$ & $\mathrm{n} / \mathrm{r}$ & 0.9999 & 0.9999 & $\mathrm{n} / \mathrm{r}$ \\
\hline SLC6A6 & rs4685154 & 0.5897 & 0.9635 & 0.6535 & 0.1191 & 0.1927 & 0.0470 & 0.2540 & 0.8530 & 0.4554 \\
\hline TNFRSF1B & rs3397 & 0.2835 & 0.0896 & 0.1402 & 0.1193 & 0.0493 & 0.5872 & 0.9999 & 0.9990 & 1.0000 \\
\hline TNFSF 15 & rs3810936 & 0.8782 & 0.5239 & 0.5199 & 0.1291 & 0.8847 & 0.0457 & 0.8960 & 0.2650 & 0.7494 \\
\hline TNFSF 15 & rs 7867918 & 0.0438 & 0.0824 & 0.1652 & 0.0613 & 0.2248 & 0.0718 & 0.0729 & 0.1870 & 0.7609 \\
\hline TNRC6C & rs 4362447 & 0.0636 & 0.1906 & 0.9391 & 0.0285 & 0.8595 & 0.0795 & 0.9970 & 0.5730 & 0.4840 \\
\hline TRAIP & rs17598137 & 0.9995 & 0.9997 & 0.9996 & 0.9996 & 0.9996 & 0.9996 & 0.9999 & 0.0951 & 0.0248 \\
\hline TRAIP & rs2271960 & 0.3267 & 0.0354 & 0.3621 & 0.3636 & 0.4224 & 0.8182 & 0.2940 & 0.1050 & 0.9996 \\
\hline TRAIP & rs6446298 & 0.0424 & 0.0556 & 0.6071 & 0.0989 & 0.1827 & 0.1409 & 0.9840 & 0.2270 & 0.9997 \\
\hline USP4 & rs9881860 & 0.8590 & 0.3827 & 0.3214 & 0.8727 & 0.6817 & 0.9307 & 0.8077 & 0.0449 & 0.2229 \\
\hline
\end{tabular}

Table 4. Results of SNPs from candidate genes associated with adverse effects of Brassicaceae; showing $p$-values only; significant $p$ values highlighted in italic and bold. (* Remained significant after applying multiple testing correction using false discovery rate).

\begin{tabular}{|c|c|c|c|c|c|c|c|c|c|c|c|}
\hline Gene & SNP & Broccoli & Cabbage & Cauliflower & $\begin{array}{c}\text { Chinese } \\
\text { Greens }\end{array}$ & Rocket & Watercress & Horseradish & $\begin{array}{c}\text { Mustard } \\
\text { powder }\end{array}$ & $\begin{array}{c}\text { Mustard } \\
\text { Sauce } \\
\end{array}$ & Wasabi \\
\hline AMT & rs1464567 & 0.0652 & 0.9368 & 0.0667 & 0.0376 & 0.2402 & 0.2171 & 0.9354 & 0.4783 & 0.8241 & 0.5862 \\
\hline ATG16L1 & rs 10210302 & 0.7140 & 0.0232 & 0.2158 & 0.0559 & 0.3950 & 0.3398 & 0.2528 & 0.4525 & 0.0537 & 0.3769 \\
\hline BSN & rs2131109 & 0.3418 & 0.5838 & 0.0317 & 0.6593 & 0.4865 & 0.4534 & 0.5293 & 0.5810 & 0.5959 & 0.6147 \\
\hline BSN & rs4283605 & 0.7291 & 0.1096 & 0.7757 & 0.8219 & 0.5448 & 0.6208 & 0.0235 & 0.9420 & 0.7979 & 0.4625 \\
\hline CDH29 & rs7629936 & 0.1395 & 0.7087 & 0.0494 & 0.1481 & 0.2022 & 0.2251 & 0.5519 & 0.5841 & 0.9684 & 0.0803 \\
\hline CDKAL1 & rs6908425 & 0.8750 & 0.6255 & 0.8891 & 0.8246 & 0.2999 & 0.0298 & 0.2657 & 0.7070 & 0.3470 & 0.5407 \\
\hline CLDN12 & rs1017106 & 0.8662 & 0.0291 & 0.3861 & 0.9996 & 0.9996 & 0.9900 & 0.0534 & 0.3336 & 0.0812 & 0.0527 \\
\hline CLDN12 & rs 17864006 & 0.3710 & 0.1913 & 0.3723 & 0.0312 & 0.2893 & 0.8806 & 0.8001 & 0.5230 & 0.9520 & 0.8456 \\
\hline CLDN2 & rs 12008279 & 0.4042 & 0.8127 & 0.8816 & 0.1002 & 0.0481 & 0.8982 & 0.3240 & 0.8170 & 0.8822 & 0.5584 \\
\hline
\end{tabular}


Table 4. Cont.

\begin{tabular}{|c|c|c|c|c|c|c|c|c|c|c|c|}
\hline desert_PTGER4 & rs17234657 & 0.3319 & 0.0458 & 0.6535 & 0.0672 & 0.8378 & 0.9958 & 0.0959 & 0.0244 & 0.6530 & 0.1830 \\
\hline DIO1 & rs11206244 & 0.3296 & 0.0968 & 0.4987 & 0.2154 & 0.2448 & 0.5280 & 0.0136 & 0.3460 & 0.1520 & 0.4456 \\
\hline DIO1 & rs7515322 & $0.000167 *$ & 0.1070 & 0.0864 & 0.5197 & 0.2208 & 0.0512 & 0.6658 & 0.3440 & 0.6550 & 0.3161 \\
\hline $\mathrm{DIO} 2$ & rs 12885300 & 0.2305 & 0.7110 & 0.9809 & 0.2811 & 0.5710 & 0.5532 & 0.0083 & 0.0833 & 0.2000 & 0.1935 \\
\hline $\mathrm{DIO} 3$ & rs1190715 & 0.0252 & 0.8963 & 0.6316 & 0.6761 & 0.9875 & 0.5535 & 0.2095 & 0.5380 & 0.5386 & 0.0211 \\
\hline $\mathrm{DIO} 3$ & rs1190716 & 0.1362 & 0.1126 & 0.9806 & 0.3182 & 0.5579 & 0.0361 & 0.1060 & 0.2720 & 0.2530 & 0.0039 \\
\hline DIO3 & rs945006 & 0.5967 & 0.2792 & 0.2923 & 0.7176 & 0.6002 & 0.9202 & 0.0791 & 0.2230 & 0.1980 & 0.0019 \\
\hline DLG5 & rs2289311 & 0.3003 & 0.0830 & 0.8446 & 0.7854 & 0.8730 & 0.0423 & 0.2711 & 0.1390 & 0.7660 & 0.5258 \\
\hline FHIT & rs2250114 & 0.3770 & 0.4506 & 0.4490 & 0.0066 & 0.8620 & 0.4285 & 0.1317 & 0.9320 & 0.5110 & 0.0191 \\
\hline GPX1 & rs1800668 & 0.5773 & 0.7500 & 0.7313 & 0.6076 & 0.2872 & 0.2612 & 0.0418 & 0.1677 & 0.4277 & 0.1434 \\
\hline GPX2 & rs1800669 & 0.3562 & 0.0374 & 0.2652 & 0.3266 & 0.9201 & 0.9997 & 0.3097 & 0.1110 & 0.0830 & 0.7837 \\
\hline GPX2 & rs 2296327 & 0.6288 & 0.8722 & 0.6316 & 0.4766 & 0.1252 & 0.8774 & 0.3734 & 0.8050 & 0.8450 & 0.0392 \\
\hline GPX2 & rs2412065 & 0.6747 & 0.8985 & 0.6886 & 0.7937 & 0.9786 & 0.7360 & 0.0807 & 0.0377 & 0.0101 & 0.0164 \\
\hline GPX2 & rs2737844 & 0.5789 & 0.6613 & 0.2104 & 0.2119 & 0.2871 & 0.4791 & 0.2838 & 0.0637 & 0.1543 & 0.0230 \\
\hline GPX2 & rs3742599 & 0.5281 & 0.7762 & 0.8361 & 0.5321 & 0.7983 & 0.7495 & 0.1121 & 0.1326 & 0.0673 & 0.0276 \\
\hline GPX3 & rs2042235 & 0.3841 & 0.5588 & 0.0719 & 0.3311 & 0.0019 & 0.0958 & 0.4810 & 0.2850 & 0.5060 & 0.1288 \\
\hline GPX3 & rs3792796 & 0.6028 & 0.5677 & 0.7284 & 0.7336 & 0.1833 & 0.4795 & 0.8515 & 0.0202 & 0.3904 & 0.7421 \\
\hline GPX3 & rs3792797 & 0.8569 & 0.6572 & 0.9486 & 0.4927 & 0.0135 & 0.0546 & 0.8075 & 0.3264 & 0.9458 & 0.2587 \\
\hline GPX3 & rs3805435 & 0.0355 & 0.3907 & 0.2392 & 1.0000 & 0.0251 & 0.0996 & 0.1762 & 0.5750 & 0.8380 & 0.1363 \\
\hline GPX3 & rs3828599 & 0.6441 & 0.1837 & 0.6508 & 0.7001 & 0.0368 & 0.1743 & 0.7133 & 0.0674 & 0.7072 & 0.3305 \\
\hline GPX3 & rs8177425 & 0.0300 & 0.6436 & 0.0040 & 0.1006 & 0.2489 & 0.4742 & 0.8150 & 0.9999 & 0.9999 & 0.3026 \\
\hline HLA & rs 9469220 & 0.5098 & 0.3760 & 0.4180 & 0.2910 & $0.0000751 *$ & 0.2241 & 0.2165 & 0.6360 & 0.5656 & 0.4644 \\
\hline IBD5 & rs 10077785 & 0.6067 & 0.6985 & 0.3401 & 0.9251 & 0.2185 & 0.0447 & 0.7456 & 0.7740 & 0.9120 & 0.8265 \\
\hline IL12B & rs6887695 & 0.7405 & 0.0399 & 0.5853 & 0.0159 & 0.4712 & 0.5117 & 0.0807 & 0.2417 & 0.0309 & 0.0131 \\
\hline IL23R & rs 11209026 & 0.0239 & 0.1584 & 0.5010 & 0.3494 & 0.4815 & 0.8682 & 0.5304 & 0.4450 & 0.8000 & 0.7140 \\
\hline IL23R & rs 7517847 & 0.1036 & 0.0194 & 0.0845 & 0.0040 & 0.8793 & 0.0253 & 0.2731 & 0.3032 & 0.6869 & 0.9516 \\
\hline JAK2 & rs 10758669 & 0.6194 & 0.5804 & 0.0112 & 0.5926 & 0.6808 & 0.1891 & 0.4730 & 0.3900 & 0.9834 & 0.4026 \\
\hline JAM3 & rs 11604455 & 0.2993 & 0.7612 & 0.3313 & 0.7678 & 0.0172 & 0.9997 & 0.2734 & 0.8570 & 0.2700 & 0.9695 \\
\hline LAMP1 & rs 12871648 & 0.1360 & 0.8643 & 0.0154 & 0.4294 & 0.8006 & 1.0000 & 0.5318 & 0.4350 & 0.2880 & 0.2019 \\
\hline
\end{tabular}


Table 4. Cont.

\begin{tabular}{|c|c|c|c|c|c|c|c|c|c|c|c|}
\hline MAP1LC3B & rs8051218 & 0.8073 & 0.0473 & 0.4587 & 0.4253 & 0.9996 & 0.0709 & 0.0687 & 0.4940 & 0.4480 & 0.3652 \\
\hline NOD2 & rs2066844 & 0.1270 & 0.8670 & 0.0484 & 0.4170 & 0.9760 & 0.1940 & 0.7067 & 0.0463 & 0.1670 & 0.7287 \\
\hline OCTN1 & rs1050152 & 0.0028 & 0.0630 & 0.0167 & 0.1174 & 0.2692 & 0.9600 & 0.0422 & 0.0714 & 0.1230 & 0.0691 \\
\hline OCTN2 & rs2631367 & 0.0026 & 0.1222 & 0.0071 & 0.0862 & 0.4066 & 0.7919 & 0.0273 & 0.0322 & 0.1665 & 0.0317 \\
\hline P4HA2 & rs4361509 & 0.1683 & 0.2970 & 0.2569 & 0.0796 & 0.0367 & 0.2332 & 0.5564 & 0.1805 & 0.2497 & 0.0298 \\
\hline PTGER4 & rs 4613763 & 0.2721 & 0.0752 & 0.6681 & 0.0364 & 0.5922 & 0.5215 & 0.1237 & 0.0210 & 0.6250 & 0.4243 \\
\hline SELS & rs4965814 & 0.0348 & 0.0326 & 0.8601 & 0.6410 & 0.1583 & 0.3080 & 0.0646 & 0.6777 & 0.7620 & 0.8457 \\
\hline SELS & rs7178239 & 0.0329 & 0.0219 & 0.5408 & 0.9585 & 0.2971 & 0.1962 & 0.1168 & 0.5900 & 0.8680 & 0.9406 \\
\hline SEP_15 & rs5845 & 0.4563 & 0.0232 & 0.9003 & 0.2900 & 0.6073 & 0.0475 & 0.5742 & 0.3418 & 0.0692 & 0.8078 \\
\hline SEP_15 & rs5859 & 0.4050 & 0.0149 & 0.8041 & 0.2895 & 0.5395 & 0.0287 & 0.2979 & 0.3980 & 0.0511 & 0.4922 \\
\hline SLC11A1 & rs3731865 & 0.7790 & 0.7499 & 0.0302 & 0.5538 & 0.9035 & 0.8647 & 0.6170 & 0.7168 & 0.4885 & 0.5708 \\
\hline SLC11A2 & rs224589 & 0.1352 & 0.2771 & 0.3233 & 0.5982 & 0.0433 & 0.0184 & 0.2881 & 0.1440 & 0.5550 & 0.4478 \\
\hline SLC11A2 & rs 427020 & 0.6998 & 0.7563 & 0.0494 & 0.8253 & 0.0098 & 0.0772 & 0.2422 & 0.6477 & 0.8684 & 0.0282 \\
\hline SLC6A6 & rs 4685154 & 0.9413 & 0.3352 & 0.7719 & 0.0359 & 0.4094 & 0.6288 & 0.9841 & 0.5260 & 0.9491 & 0.3369 \\
\hline STAT3 & rs744166 & 0.5240 & 0.0327 & 0.2239 & 0.2932 & 0.9202 & 0.4768 & 0.5676 & 0.6510 & 0.2256 & 0.6283 \\
\hline $\mathrm{TF}$ & rs1799899 & 0.0269 & 0.4295 & 0.6292 & 0.7491 & 0.8285 & 0.6787 & 0.6008 & 0.7040 & 0.5270 & 0.5817 \\
\hline TFF3 & rs225369 & 0.1607 & 0.3494 & 0.0171 & 0.9542 & 0.8237 & 0.8419 & 0.8338 & 0.1928 & 0.7704 & 0.3020 \\
\hline TLR9 & rs5743836 & 0.5591 & 0.0495 & 0.6591 & 0.3329 & 0.3057 & 0.4265 & 0.5340 & 0.9000 & 0.6370 & 0.2379 \\
\hline TNF.ALPHA & rs1800629 & 0.0391 & 0.8413 & 0.3526 & 0.3925 & 0.3941 & 0.2303 & 0.9387 & 0.2080 & 0.3790 & 0.1965 \\
\hline TNFSF15 & rs10982412 & 0.8758 & 0.6216 & 0.7821 & 0.7470 & 0.6171 & 0.0288 & 0.9820 & 0.7528 & 0.2133 & 0.7304 \\
\hline TNFSF15 & rs7029554 & 0.6069 & 0.7134 & 0.7987 & 0.8799 & 0.5054 & 0.0272 & 0.5918 & 0.5770 & 0.0482 & 0.4466 \\
\hline TRAIP & rs 10865959 & 0.0194 & 0.3572 & 0.0580 & 0.3637 & 0.0823 & 0.4026 & 0.5807 & 0.9487 & 0.9680 & 0.0578 \\
\hline TRAIP & rs 17598137 & 0.0194 & 0.3766 & 0.0152 & 0.7812 & 0.6667 & 0.2056 & 0.3028 & 0.5790 & 0.6020 & 0.4352 \\
\hline USP4 & rs1865741 & 0.1737 & 0.2335 & 0.0663 & 0.0337 & 0.3255 & 0.6525 & 0.1860 & 0.6360 & 0.8208 & 0.6487 \\
\hline USP4 & rs9881860 & 0.0232 & 0.1275 & 0.0474 & 0.0657 & 0.9879 & 0.8338 & 0.0635 & 0.7669 & 0.8724 & 0.4960 \\
\hline VDR & rs7975232 & 0.8557 & 0.5518 & 0.6355 & 0.6789 & 0.1431 & 0.4863 & 0.0488 & 0.6940 & 0.3780 & 0.4483 \\
\hline
\end{tabular}




\section{Discussion}

On looking more closely at the candidate SNPs associated with CD that were tested for beneficial or adverse effects with the ingestion of selected Brassicaceae, we noted, as one would expect with an inflammatory disorder like Crohn's disease that several of the genes associated with these SNPs were involved in functions relating to immunity. AGT12 (rs26532) and AGT16L1 (rs10210302) are necessary for autophagy; CSFIR (rs2282804), governs macrophages, DEFA6 (rs712276) relates to the Paneth cells and its role in defence and HLA (rs9469220) a major histocompatibility complex, also involves the immune system. The ICAMIgene, (rs1799969) is expressed on the cells of the immune system and the endothelium [27]. IL12B, (rs6887695), has also been associated with another immune disorder, asthma [28]. IRGM, (rs4958847) is a gene associated with the autophagy pathway in CD [29]. IL23R, (rs11209026 and rs7517847) is another gene recognised as being involved in the adaptive immunity pathway as is $J A K 2$ ( $\mathrm{rs} 10758669)$ which is also essential for signalling events in innate immunity [28]. PTPN2 (rs2542151) controls a range of cellular processes e.g., cell growth and TLR9 (rs5743836) has a major role in both innate and adaptive immunity [28].

A number of SNPs are also associated with genes involved in cell transport, rs 1050152 (SLC22A4/OCTN1) and rs2631367 (SLC22A5/OCTN2) linked to carnitine [30-32]; rs224589 and rs427020 (SLC11A2) linked to iron. ITLN1 (rs2274910) and TF (rs1799899) are other genes associated with iron metabolism [28]. The SNPs rs41284011 and rs4685154 (SLC6A6) are linked with taurine which is linked to muscle function and having $\mathrm{CD}$ has been linked to diminished muscle strength [33-35]. Another gene of interest is the VDR gene (rs7975232) which facilitates the action of vitamin D3, as well as having a role in the homeostasis of calcium [36]. CDH29 (rs7629936) is also a gene, with a SNP identified in this analysis, linked to calcium via calcium-reliant cell adhesion proteins [28]. Other genes of note are associated with responses of people with CD eating Brassicaceae are the DIO1 (rs11206244 and rs75153322) and DIO3 (rs1190715, rs1190716, and rs945006) genes which are important for the proper functioning of the thyroid gland. DIO1, also encodes selenoproteins [37,38] as does GPX3, (rs2042235, rs3763013, rs3792796, rs3792797, rs3805435, rs3828599, rs8177425, and rs870407, GPX1 (rs1800668) and GPX2 (rs1800669, rs2296327, rs2412065, rs2737844, rs37425990).

It is curious to note that six of the candidate genes identified have SNPS that are linked with both adverse and beneficial effects. The SNP rs7629936 (CDH29) is associated with adverse effects for cauliflower and beneficial effects for cabbage. The SNP rs2250114 (FHIT) is associated with adverse effects to Chinese greens and wasabi but beneficial effects for mustard powder and mustard sauce. The SNP rs3792796 (GPX3) is associated with adverse effects to mustard powder with beneficial effects to cauliflower and broccoli. The SNP rs2066844 (NOD2) is associated with adverse effects to cauliflower and mustard sauce and beneficial effects to mustard powder. The SNP rs5859 (SEP15) is associated with adverse effects to cabbage and watercress but beneficial effects to mustard powder. The SNP rs9881860 (USP4) is associated with adverse effects to broccoli and Chinese greens and beneficial effects to mustard sauce.

These differences in tolerance may relate to the different composition of each Brassicaceae. Brassicaceae differ in their nutritional components especially with respect to their phytochemical composition [8]. They are also unique in that they contain the phytochemicals known as 
glucosinolates. Up to two hundred different glucosinolates have been recorded [39]. These have been intensely researched because of their links with cancer reduction and properties associated with destroying or inhibiting the growth of bacteria, fungi and nematodes [40]. Each plant species of the Brassicaceae family may also contain up to four different classes of glucosinolates. The most common glucosinolates in cabbage are for example glucobrassicin, glucoraphanin, glucotropaeolin and sinigrin whereas in broccoli the abundant glucosinolates are glucoraphanin, glucobrassicin, gluconapin, and progoitrin [41]. Rocket's predominate glucosinolates are glucoraphanin, DMB-GLS and glucoerucin [42].

When the vegetables are cooked, the glucosinolates reach the large intestine intact and the microbiota release the isothiocyanates and other metabolites with diverse outcomes [43]. The different combinations of glucosinolates in combination with particular SNPs may contribute to dissimilar outcomes produced by the microbiota. People with Crohn's disease also have a microbiota community that differs from those with a normal gut [44]. So these differences too in the microbiota communities will also play a part in the difference in response. Escherichia coli are bacteria which have been identified as having increased numbers in those with Crohn's disease [45-48]. They have also been associated with formation of granuloma (a common feature of Crohn's disease) when internalised by macrophages in vitro [49]. Escherichia coli movement across Membranous cells (M-cells) has been shown in vitro to be reduced by soluble plant fibres such as broccoli [50]. [M-cells are part of the epithelium on the lymphoid follicles of the large intestine and act as a portal by which microorganisms can gain entry] [51].The ingestion of broccoli has also been shown in the IBD mouse model mdrla(-/-) to lower inflammation in the large bowel through changes in the microbiota metabolism [52]. However, from the analysis provided in this paper, this response maybe modulated in a different way in humans depending on the particular SNP they have. For example the SNP rs9469220 variant in HLA and the SNP rs7515322 in DIO1 which remained significant after correcting for multiple testing.

The rs9469220 variant in $H L A$ was associated with an adverse reaction to the Brassicaceae rocket. HLA (human leucocyte antigen) genes are located in the region of MHCII in chromosome 6. This is very close to the HLA complexP5 (HCP5) which is associated with susceptibility to autoimmune diseases [53]. Variations in the HLA region have been associated with an inflammatory colonic phenotype [54-56]. HLA contains 4 classes and this SNP is with the class II MHC subgroup DO alpha. Both Class I and II HLA genes are necessary for normal lymphocyte performance [56]. This subgroup is associated with extracellular proteins that regulate peptide loading with antigens [57,58]. Class II molecules engage with CD4 $\mathrm{T}$ cells. These activate an immune response which may involve inflammation with the enlisting of phagocytes or activate B cells engaging antibodies in the immune response [59,60]. The polymorphism rs9469220 (identified in this study with an adverse response to rocket) has been linked to total IgE levels $[61,62]$. IgE has a pivotal role in type I sensitivity associated with allergic forms of asthma, rhinitis, urticaria and dermatitis [63]. IgE is also recognized as providing immunity to parasites [64]. The fact that the SNP rs9469220, in people with CD in this study is significantly associated with an adverse reaction to the Brassicaceae rocket would suggest that ingestion of this food engages the immune response in a type I sensitivity reaction. Hence an exacerbation of symptoms of CD occurs.

The other SNP which remained significant after correcting for multiple testing, was SNP (rs7515322) in DIO1. DIO1 is part of the family of selenoenzymes which are important signalling molecules which activate or deactivate thyroid hormones, so play a key role in thyroid metabolism. As 
thyroid hormones are key to the development and metabolism of most tissues, these selenoproteins have a significant contribution to make. The DIO1 gene is located in chromosome 1p33-p32 [28]. It is described as a thioredoxin fold integral membrane protein found in the plasma membrane. Transcripts of it have been identified in the intestines, thyroid, gonads, pituitary gland and placenta [65]. DIOI has a number of roles. It supplies a large portion of circulating plasma $T_{3}$, it also acts as a scavenging enzyme removing inactive iodothyronines and recycling iodine, as well as playing a part in thyronamine biosynthesis [66-68]. Studies have also shown DIO activity associated with local inflammation and tumoural tissues [69,70]. DIO1 as a selenoprotein has also been discovered to be protective against iodine deficiency when its activity is reduced with selenium deficiency. This was observed in regions of endemic goitre, when selenium was given to people with iodine deficiency with a consequent lowering of thyroid function [71]. Selenium levels have been reported as being significantly lower in New Zealand people with Crohn's disease [72]. People in New Zealand with Crohn's disease and low selenium levels and with this SNP rs7515322 may have also a heightened adverse response to Broccoli.

However, another mechanism may be involved. Critically ill people have been shown to have lower DIO1 hepatic activity, and this has been thought to be modulated through Cytokines e.g., ILI, IL6 and $T N F \alpha$ [73]. Cytokines may also decrease the function of $D I O 1$ and $I L-1 \beta$ has been shown to inhibit $D I O 1$ activities in hepatocarcinoma cell lines [74]. $I L-1 \beta$ is one of the cytokines increased in patients with Crohn's disease [75] and associated with inflammation in this disorder. This SNP rs9469220 could link to an adverse response through progoitrin one of the most abundant glucosinolates in broccoli [41]. Progoitrin after ingestion is converted to goitrin by the activity in bacteria [76] and goitrin is known to decrease thyroid hormone production [77].

In addition, many adults in NZ are in a state of mild iodine deficiency with the adult population having a medium iodine concentration of $53 \mu \mathrm{g} / \mathrm{L}$ [78]. Schneider et al. in their murine study showed that DIO1 may enable the major thyroid hormone $\mathrm{T}_{3}$ to be released into the circulatory system when an animal was iodine deficient [66]. Thyrocytes also react quickly to iodine deficiency by increasing the release of angiogenic signals. This is a VEGF-A dependent process [79]. VEGF production has been shown to be significantly increased with active Crohn's disease [80].

Those people with CD having this SNP rs7515322 may with the ingestion of broccoli experience a greater degree of deficiency in iodine. This combined with a decrease in DIOI activity from the influence of IL-1beta activity associated with CD (DIOI normally increases with iodine deficiency) as well as an increased VEGF levels with the release of angiogenic signals with thyrocyte production, may exacerbate their symptoms of CD.

When investigating individual SNPs from this analysis of a New Zealand population with CD, it is interesting to note the two SNPs which are significantly associated with adverse effects (after correcting for multiple testing) to the two Brassicaceae-broccoli and rocket. Studying a wider range of Brassicaceae would increase the possibility of the identification of more significant SNPs and improve the range of Brassicaceae that would be available to choose from. If people want to avoid the long and tedious trial and error approach to working out what they can eat, or be more discerning about advice given to avoid many of the Brassicaceae as in the FODMAP diet [81] knowledge of their SNP profile and how it interacts with Brassicaceae would be helpful. It would enable people to select Brassicaceae more appropriately and maximise on their nutritional benefits. 


\section{Conclusions}

It is now possible to identify two specific SNPs rs7515322 (DIO1), and rs9469220 (HLA) associated with the two forms of Brassicaceae broccoli and rocket respectively, with regard to having an adverse effect on the symptoms of people with $\mathrm{CD}$. Further research is required to substantiate our findings and to conclusively determine the nature of the observed association of an adverse effect with the two forms of Brassicaceae broccoli and rocket and the two SNPs rs7515322 (DIO1), and rs9469220 (HLA) respectively, with CD. This study indicates that the tolerance of some varieties of Brassicaceae may be predicted by analysis of a person's genotype. This information is a step towards enabling those with $\mathrm{CD}$ to select appropriate Brassicaceae and be exposed to key nutrients. This raises the possibility that with suitable nutrition, there is a real prospect of a significant improvement in symptoms associated with this disorder.

\section{Acknowledgements}

This analysis was based on the secondary analysis of data from the "Genes and Diet in IBD Study". The authors of this original data set were Angharad R Morgan, Wen Jiun Lam, Christopher M Triggs and Alan G Fraser. Nutrigenomics New Zealand is a collaboration between AgResearch Ltd., Plant and Food Research and The University of Auckland, with funding through the Ministry of Business, Innovation and Employment (MBIE). This particular study was possible through the financial support from the Valrae Collins' scholarship for research on CD.

\section{Conflicts of Interest}

The authors declare no conflicts of interest.

\section{References}

1. Gearry, R.B.; Richardson, A.; Frampton, C.M.A.; Collett, J.A.; Burt, M.J.; Chapman, B.A.; Barclay, M.L. High incidence of Crohn's disease in Canterbury, New Zealand: Results of an epidemiologic study. Inflamm. Bowel Dis. 2006, 12, 936-943.

2. Han, D.Y.; Fraser, A.G.; Dryland, P.; Ferguson, L.R. Environmental factors in the development of chronic inflammation: A case-control study on risk factors for Crohn's disease within New Zealand. Mutat. Res. 2010, 690, 116-122.

3. Tsironi, E.; Feakins, R.M.; Roberts, C.S.; Rampton, D.S. Incidence of inflammatory bowel disease is rising and abdominal tuberculosis is falling in Bangladeshis in East London, United Kingdom. Am. J. Gastroenterol. 2004, 99, 1749-1755.

4. Triggs, C.M.; Munday, K.; Hu, R.; Fraser, A.G.; Gearry, R.B.; Barclay, M.L.; Ferguson, L.R. Dietary factors in chronic inflammation: Food tolerances and intolerances of a New Zealand Caucasian Crohn's disease population. Mutat. Res. 2010, 690, 123-138.

5. Barnett, M.; Bermingham, E.; McNabb, W.; Bassett, S.; Armstrong, K.; Rounce, J.; Roy, N. Investigating micronutrients and epigenetic mechanisms in relation to inflammatory bowel disease. Mutat. Res. 2010, 690, 71-80. 
6. Ballegaard, M.; Bjergstrøm, A.; Brøndum, S.; Hylander, E.; Jensen, L.; Ladefoged, K. Self-reported food intolerance in chronic inflammatory bowel disease. Scand. J. Gastroenterol. 1997, 32, 569-571.

7. Van den Bogaerde, J.; Kamm, M.A.; Knight, S.C. Immune sensitization to food, yeast and bacteria in Crohn's disease. Aliment. Pharmacol. Ther. 2001, 15, 1647-1653.

8. Campbell, B.; Han, D.Y.; Triggs, C.M.; Fraser, A.G.; Ferguson, L.R. Brassicaceae: Nutrient analysis and investigation of tolerability in people with Crohn's disease in a New Zealand study. Funct. Foods Health Dis. 2012, 2, 460-486.

9. Duke, J.; Beckstrom-Sternberg, S. Phytochemical Database USDA-ARS-NGRL. Beltsbille Agricultural Research Center. Beltsbille, Maryland. Available online: http://www.ars.grin.gov/ cgl-bin/duke/ethnobot.pl (accessed on 23 August 2011).

10. Gebhardt, S.; Lemar, L.; Haytowitz, D.; Pehrsson, P.; Nickle, M.; Showell, B.; Thomas, R.; Exler, J.; Holden, J. USDA National Nutrient Database for Standard Reference, Release 21. 2008. Available online: http://www.ars.usda.gov/main/site_main.htm?modecode=12-35-45-00 (accessed on 24 March 2010).

11. Burlingame, B.A.; Milligan, G.; Apimerika, D.; Arthur, J. The Concise New Zealand Food Composition Tables, 8th ed.; New Zealand Institute for Crop \& Food Research: Wellington, New Zealand, 2009; pp. 97-108.

12. Shils, M.E.; Shike, M. Modern Nutrition in Health and Diseases; Lippincott Williams \& Wilkins: Philadelphia, PA, USA, 2006; pp. 413-414.

13. Alberg, A.J. The influence of cigarette smoking on circulating concentrations of antioxidant micronutrients. Toxicology 2002, 180, 121-137.

14. Solomons, N.; Rosenberg, I.; Sandstead, H.; Vo-Khactu, K. Zinc deficiency in Crohn's disease. Digestion 1977, 16, 87-95.

15. Wible, B.A.; Wang, L.; Kuryshev, Y.A.; Basu, A.; Haldar, S.; Brown, A.M. Increased K efflux and apoptosis induced by the potassium channel modulatory protein KChAP/PIAS3 $\beta$ in prostate cancer cells. J. Biol. Chem. 2002, 277, 17852-17862.

16. Bakker, S.F.; Dik, V.K.; Witte, B.I.; Lips, P.; Roos, J.C.; van Bodegraven, A.A. Increase in bone mineral density in strictly treated Crohn's disease patients with concomitant calcium and vitamin D supplementation. J. Crohn's Colitis 2013, 7, 377-384.

17. Carmel, R.; Green, R.; Rosenblatt, D.S.; Watkins, D. Update on cobalamin, folate, and homocysteine. ASH Educ. Program Book 2003, 2003, 62-81.

18. Conterno, L.; Fava, F.; Viola, R.; Tuohy, K.M. Obesity and the gut microbiota: Does up-regulating colonic fermentation protect against obesity and metabolic disease? Genes Nutr. 2011, 6, 241-260.

19. Fotsis, T.; Pepper, M.S.; Montesano, R.; Aktas, E.; Breit, S.; Schweigerer, L.; Rasku, S.; Wähälä, K.; Adlercreutz, H. Phytoestrogens and inhibition of angiogenesis. Baillière's Clin. Endocrinol. Metab. 1998, 12, 649-666.

20. Karunasinghe, N.; Ryan, J.; Tuckey, J.; Masters, J.; Jamieson, M.; Clarke, L.C.; Marshall, J.R.; Ferguson, L.R. DNA stability and serum selenium levels in a high-risk group for prostate cancer. Cancer Epidemiol. Biomark. Prev. 2004, 13, 391-397.

21. Parl, F.F. Glutathione S-transferase genotypes and cancer risk. Cancer Lett. 2005, 221, 123-129. 
22. Capra, S. Nutrient Reference Values for Australia and New Zealand: Including Recommended Dietary Intakes; Commonwealth of Australia: Canberra, ACT, Australia, 2006; pp. 21-77.

23. Joachim, G. Responses of people with inflammatory bowel disease to foods consumed. Gastroenterol. Nurs. 2000, 23, 160-167.

24. Satsangi, J.; Silverberg, M.; Vermeire, S.; Colombel, J. The Montreal classification of inflammatory bowel disease: Controversies, consensus, and implications. Gut 2006, 55, 749-753.

25. Storey, J.D. A direct approach to false discovery rates. J. R. Stat. Soc. Series B (Stat. Methodol.) 2002, 64, 479-498.

26. Team, R.C. R: A Language and Environment for Statistical Computing; R Foundation for Statistical Computing: Vienna, Austria, 2013.

27. Rothlein, R.; Dustin, M.; Marlin, S.; Springer, T. A human intercellular adhesion molecule (ICAM-1) distinct from LFA-1. J. Immunol. 1986, 137, 1270-1274.

28. Rebhan, M.; Chalifa-Caspi, V.; Prilusky, J.; Lancet, D. GeneCards: A novel functional genomics compendium with automated data mining and query reformulation support. Bioinformatics 1998, 14, 656-664.

29. Lees, C.; Barrett, J.; Parkes, M.; Satsangi, J. New IBD genetics: Common pathways with other diseases. Gut 2011, 60, 1739-1753.

30. Flanagan, J.L.; Simmons, P.A.; Vehige, J.; Willcox, M.D.; Garrett, Q. Review role of carnitine in disease. Nutr. Metab. 2010, 7, 30.

31. Steiber, A.; Kerner, J.; Hoppel, C.L. Carnitine: A nutritional, biosynthetic, and functional perspective. Mol. Asp. Med. 2004, 25, 455-473.

32. Benvenga, S.; Amato, A.; Calvani, M.; Trimarchi, F. Effects of carnitine on thyroid hormone action. Ann. N. Y. Acad. Sci. 2004, 1033, 158-167.

33. Warskulat, U.; Flögel, U.; Jacoby, C.; Hartwig, H.; Thewissen, M.; Merx, M.W.; Molojavyi, A.; Heller-Stilb, B.; Schrader, J.; Häussinger, D. Taurine transporter knockout depletes muscle taurine levels and results in severe skeletal muscle impairment but leaves cardiac function uncompromised. FASEB J. 2004, 18, 577-579.

34. Stapleton, P.P.; O'Flaherty, L.; Redmond, H.P.; Bouchier-Hayes, D.J. Review: Host defense-A role for the amino acid taurine? J. Parenter. Enter. Nutr. 1998, 22, 42-48.

35. Valentini, L.; Schaper, L.; Buning, C.; Hengstermann, S.; Koernicke, T.; Tillinger, W.; Guglielmi, F.W.; Norman, K.; Buhner, S.; Ockenga, J. Malnutrition and impaired muscle strength in patients with Crohn's disease and ulcerative colitis in remission. Nutrition 2008, 24, 694-702.

36. Raftery, T.; O’Morain, C.A.; OSullivan, M. Vitamin D: New roles and therapeutic potential in inflammatory bowel disease. Curr. Drug Metab. 2012, 13, 1294-1302.

37. Papp, L.V.; Lu, J.; Holmgren, A.; Khanna, K.K. From selenium to selenoproteins: Synthesis, identity, and their role in human health. Antioxid. Redox Signal. 2007, 9, 775-806.

38. Peeters, R.P.; van der Deure, W.M.; Visser, T.J. Genetic variation in thyroid hormone pathway genes; polymorphisms in the TSH receptor and the iodothyronine deiodinases. Eur. J. Endocrinol. 2006, 155, 655-662.

39. Clarke, D.B. Glucosinolates, structures and analysis in food. Anal. Methods 2010, 2, 310-325.

40. Fahey, J.W.; Zalcmann, A.T.; Talalay, P. The chemical diversity and distribution of glucosinolates and isothiocyanates among plants. Phytochemistry 2001, 56, 5-51. 
41. Higdon, J.V.; Delage, B.; Williams, D.E.; Dashwood, R.H. Cruciferous vegetables and human cancer risk: Epidemiologic evidence and mechanistic basis. Pharmacol. Res. 2007, 55, 224-236.

42. Pasini, F.; Verardo, V.; Caboni, M.F.; D’Antuono, L.F. Determination of glucosinolates and phenolic compounds in rocket salad by HPLC-DAD-MS: Evaluation of Eruca sativa Mill. and Diplotaxis tenuifolia L. genetic resources. Food Chem. 2012, 133, 1025-1033.

43. Mayo, B.; van Sinderen, D. Bifidobacteria: Genomics and Molecular Aspects; Horizon Scientific Press: Norfolk, UK, 2010; p. 107.

44. Nagalingam, N.A.; Lynch, S.V. Role of the microbiota in inflammatory bowel diseases. Inflamm. Bowel Dis. 2012, 18, 968-984.

45. Knight, P.; Campbell, B.J.; Rhodes, J.M. Host-bacteria interaction in inflammatory bowel disease. Br. Med. Bull. 2008, 88, 95-113.

46. Martin, H.M.; Campbell, B.J.; Hart, C.A.; Mpofu, C.; Nayar, M.; Singh, R.; Englyst, H.; Williams, H.F.; Rhodes, J.M. Enhanced Escherichia coli adherence and invasion in Crohn's disease and colon cancer. Gastroenterology 2004, 127, 80-93.

47. Darfeuille-Michaud, A.; Boudeau, J.; Bulois, P.; Neut, C.; Glasser, A.; Barnich, N.; Bringer, M.; Swidsinski, A.; Beaugerie, L.; Colombel, J. High prevalence of adherent-invasive Escherichia coli associated with ileal mucosa in Crohn's disease. Gastroenterology 2004, 127, 412-421.

48. Subramanian, S.; Roberts, C.L.; Hart, C.A.; Martin, H.M.; Edwards, S.W.; Rhodes, J.M.; Campbell, B.J. Replication of colonic Crohn's disease mucosal Escherichia coli isolates within macrophages and their susceptibility to antibiotics. Antimicrob. Agents Chemother. 2008, 52, 427-434.

49. Meconi, S.; Vercellone, A.; Levillain, F.; Payré, B.; Al Saati, T.; Capilla, F.; Desreumaux, P.; Darfeuille-Michaud, A.; Altare, F. Adherent-invasive Escherichia coli isolated from Crohn's disease patients induce granulomas in vitro. Cell. Microbiol. 2007, 9, 1252-1261.

50. Roberts, C.L.; Keita, Å.V.; Duncan, S.H.; O’Kennedy, N.; Söderholm, J.D.; Rhodes, J.M.; Campbell, B.J. Translocation of Crohn's disease Escherichia coli across M-cells: Contrasting effects of soluble plant fibres and emulsifiers. Gut 2010, 59, 1331-1339.

51. Neutra, M.R.; Mantis, N.J.; Frey, A.; Giannasca, P.J. The composition and function of M cell apical membranes: Implications for microbial pathogenesis. Semin. Immunol. 1999, 11, 171-181.

52. Paturi, G.; Mandimika, T.; Butts, C.A.; Zhu, S.; Roy, N.C.; McNabb, W.C.; Ansell, J. Influence of dietary blueberry and broccoli on cecal microbiota activity and colon morphology in mdrla(-/-) mice, a model of inflammatory bowel diseases. Nutrition 2012, 28, 324-330.

53. Cooper, D.N.; Kehrer-Sawatzki, H. Exploring the potential relevance of human-specific genes to complex disease. Hum. Genomics 2011, 5, 99-107.

54. Lees, C.W.; Satsangi, J. Genetics of inflammatory bowel disease: Implications for disease pathogenesis and natural history. Expert Rev. Gastroenterol. Hepatol. 2009, 3, 513-534.

55. Annese, V.; Piepoli, A.; Latiano, A.; Lombardi, G.; Napolitano, G.; Caruso, N.; Cocchiara, E.; Accadia, L.; Perri, F.; Andriulli, A. HLA-DRB1 alleles may influence disease phenotype in patients with inflammatory bowel disease: A critical reappraisal with review of the literature. Dis. Colon Rectum 2005, 48, 57-64.

56. Ahmad, T.; Marshall, S.E.; Jewell, D. Genetics of inflammatory bowel disease: The role of the HLA complex. World J. Gastroenterol. 2006, 12, 3628-3635. 
57. Busch, R.; Rinderknecht, C.H.; Roh, S.; Lee, A.W.; Harding, J.J.; Burster, T.; Hornell, T.; Mellins, E.D. Achieving stability through editing and chaperoning: Regulation of MHC class II peptide binding and expression. Immunol. Rev. 2005, 207, 242-260.

58. Nicholson, M.J.; Moradi, B.; Seth, N.P.; Xing, X.; Cuny, G.D.; Stein, R.L.; Wucherpfennig, K.W. Small molecules that enhance the catalytic efficiency of HLA-DM. J. Immunol. 2006, 176, 4208-4220.

59. Piatier-Tonneau, D.; Gastinel, L.; Moussy, G.; Benichou, B.; Amblard, F.; Vaigot, P.; Auffray, C. Mutations in the D strand of the human CD4 V1 domain affect CD4 interactions with the human immunodeficiency virus envelope glycoprotein gp120 and HLA class II antigens similarly. Proc. Natl. Acad. Sci. USA 1991, 88, 6858-6862.

60. Fallas, J.L.; Tobin, H.M.; Lou, O.; Guo, D.; Sant'Angelo, D.B.; Denzin, L.K. Ectopic expression of HLA-DO in mouse dendritic cells diminishes MHC class II antigen presentation. J. Immunol. 2004, 173, 1549-1560.

61. Moffatt, M.F.; Gut, I.G.; Demenais, F.; Strachan, D.P.; Bouzigon, E.; Heath, S.; von Mutius, E.; Farrall, M.; Lathrop, M.; Cookson, W.O. A large-scale, consortium-based genomewide association study of asthma. N. Engl. J. Med. 2010, 363, 1211-1221.

62. Levin, A.M.; Mathias, R.A.; Huang, L.; Roth, L.A.; Daley, D.; Myers, R.A.; Himes, B.E.; Romieu, I.; Yang, M.; Eng, C. A meta-analysis of genome-wide association studies for serum total IgE in diverse study populations. J. Allergy Clin. Immunol. 2013, 131, 1176-1184.

63. Gould, H.J.; Sutton, B.J.; Beavil, A.J.; Beavil, R.L.; McCloskey, N.; Coker, H.A.; Fear, D.; Smurthwaite, L. The biology of IgE and the basis of allergic disease. Annu. Rev. Immunol. 2003, 21, 579-628.

64. Erb, K.J. Helminths, allergic disorders and IgE-mediated immune responses: Where do we stand? Eur. J. Immunol. 2007, 37, 1170-1173.

65. Callebaut, I.; Curcio-Morelli, C.; Mornon, J.; Gereben, B.; Buettner, C.; Huang, S.; Castro, B.; Fonseca, T.L.; Harney, J.W.; Larsen, P.R. The iodothyronine selenodeiodinases are thioredoxin-fold family proteins containing a glycoside hydrolase clan GH-A-like structure. J. Biol. Chem. 2003, 278, 36887-36896.

66. Schneider, M.J.; Fiering, S.N.; Thai, B.; Wu, S.; Germain, E.S.; Parlow, A.F.; Germain, D.L.S.; Galton, V.A. Targeted disruption of the type 1 selenodeiodinase gene (DIO1) results in marked changes in thyroid hormone economy in mice. Endocrinology 2006, 147, 580-589.

67. Maia, A.L.; Goemann, I.M.; Meyer, E.L.S.; Wajner, S.M. Type 1 iodothyronine deiodinase in human physiology and disease deiodinases: The balance of thyroid hormone. J. Endocrinol. 2011, 209, 283-297.

68. Scanlan, T.S.; Suchland, K.L.; Hart, M.E.; Chiellini, G.; Huang, Y.; Kruzich, P.J.; Frascarelli, S.; Crossley, D.A.; Bunzow, J.R.; Ronca-Testoni, S. 3-Iodothyronamine is an endogenous and rapid-acting derivative of thyroid hormone. Nat. Med. 2004, 10, 638-642.

69. Boelen, A.; Kwakkel, J.; Alkemade, A.; Renckens, R.; Kaptein, E.; Kuiper, G.; Wiersinga, W.; Visser, T. Induction of type 3 deiodinase activity in inflammatory cells of mice with chronic local inflammation. Endocrinology 2005, 146, 5128-5134.

70. Debski, M.G.; Pachucki, J.; Ambroziak, M.; Olszewski, W.; Bar-Andziak, E. Human breast cancer tissue expresses high level of type $15^{\prime}$-deiodinase. Thyroid 2007, 17, 3-10. 
71. Contempre, B.; Je, D.; Bebe, N.; CH, T.; Vanderpas, J. Effect of selenium supplementation in hypothyroid subjects of an iodine and selenium deficient area: The possible danger of indiscriminate supplementation of iodine-deficient subjects with selenium. J. Clin. Endocrinol. Metab. 1991, 73, 213-215.

72. Gentschew, L.; Bishop, K.S.; Han, D.Y.; Morgan, A.R.; Fraser, A.G.; Lam, W.J.; Karunasinghe, N.; Campbell, B.; Ferguson, L.R. Selenium, selenoprotein genes and Crohn's disease in a case-control population from Auckland, New Zealand. Nutrients 2012, 4, 1247-1259.

73. Chopra, I.J.; Sakane, S.; Teco, G.N.C. A study of the serum concentration of tumor necrosis factor- $\alpha$ in thyroidal and nonthyroidal illnesses. J. Clin. Endocrinol. Metab. 1991, 72, 1113-1116.

74. Jakobs, T.C.; Mentrup, B.; Schmutzler, C.; Dreher, I.; Kohrle, J. Proinflammatory cytokines inhibit the expression and function of human type I 5'-deiodinase in HepG2 hepatocarcinoma cells. Eur. J. Endocrinol. 2002, 146, 559-566.

75. Schreiber, S.; Nikolaus, S.; Hampe, J.; Hämling, J.; Koop, I.; Groessner, B.; Lochs, H.; Raedler, A. Tumour necrosis factor $\alpha$ and Interleukin $1 \beta$ in relapse of Crohn's disease. Lancet 1999, 353, 459-461.

76. Getahun, S.M.; Chung, F. Conversion of glucosinolates to isothiocyanates in humans after ingestion of cooked watercress. Cancer Epidemiol. Biomark. Prev. 1999, 8, 447-451.

77. Bradshaw, J.E.; Heaney, R.K.; Fenwick, G.R.; McNaughton, I.H. The glucosinolate content of the leaf and stem of fodder kale (Brassica oleracea L.), Rape (Brassica napus L.) and radicole (Raphanobrassica). J. Sci. Food Agric. 1983, 34, 571-575.

78. Skeaff, S.A.; McLean, R.; Mann, J.L.; Williams, S.M. The Impact of Manditory Fortification of Bread with Iodine. Ministry of Primary Industries.MPI Technical Report Paper No: 2013/025 Wellington New Zealand. Available on line: http://www.foodsafety.govt.nz/.../manditoryfortification-bread-iodine.pdf (accessed on 20 March 2013).

79. Gérard, A.; Poncin, S.; Caetano, B.; Sonveaux, P.; Audinot, J.; Feron, O.; Colin, I.M.; Soncin, F. Iodine deficiency induces a thyroid stimulating hormone-independent early phase of microvascular reshaping in the thyroid. Am. J. Pathol. 2008, 172, 748-760.

80. Griga, T.; Tromm, A.; Spranger, J.; May, B. Increased serum levels of vascular endothelial growth factor in patients with inflammatory bowel disease. Scand. J. Gastroenterol. 1998, 33, 504-508.

81. Gearry, R.B.; Irving, P.M.; Barrett, J.S.; Nathan, D.M.; Shepherd, S.J.; Gibson, P.R. Reduction of dietary poorly absorbed short-chain carbohydrates (FODMAPs) improves abdominal symptoms in patients with inflammatory bowel disease-A pilot study. J. Crohn's Colitis 2009, 3, 8-14.

(C) 2013 by the authors; licensee MDPI, Basel, Switzerland. This article is an open access article distributed under the terms and conditions of the Creative Commons Attribution license (http://creativecommons.org/licenses/by/3.0/). 